\title{
Design and Test of Grain Moisture Online Measuring System Based on Floating Ground Capacitance
}

\author{
Mai Zhiwei ${ }^{1,}$ a, Li Changyou ${ }^{2, b}$, Wang Biying ${ }^{1, c}$ \\ ${ }^{1}$ MIDEA RAC DIVISSION, Foshan 528311, China \\ ${ }^{2}$ College of Engineering, South China Agricultural University, Guangzhou 510642, China \\ am060401819@163.com, blichyx@scau.edu.cn, c279974683@qq.com
}

\begin{abstract}
Keywords: Grain, Moisture, Online measuring, Design, Test
Abstract. A new type of capacitive grain moisture on line measuring device was designed based on the measuring principle of non-contact parallel plate floating capacitor, which was suitable to continuous dryer under severe environment. The static batching measurement was used to improve the repeated accuracy of the measurement. Positioned filling device was designed to eliminate the measuring error caused by the variation of porosity and different way of filling. Wireless communication technology was adopted to achieve real time data transmission from client sensors to computer and overcome the poor drying conditions, which could affect data transmission. The relationship among moisture, temperature, and capacitance of corn was calibrated and the system was tested on a continuous dryer. The results showed that the correlation coefficient between results of oven method and results of online measuring system was 0.992, and the absolute error of measurement was less than $\pm 0.4 \%$, while the working temperature was $15 \sim 50{ }^{\circ} \mathrm{C}$, relative humidity was $80 \% \sim 100 \%$, drying temperature was $70^{\circ} \mathrm{C}$, and the corn moisture content range was $14 \% \sim 21 \%$. It proved that the online measuring system meet the design requirement of poor drying conditions.
\end{abstract}

\section{Introduction}

Study on the analytical model of grain drying theory and control technology, it reveals the grain moisture binding energy and thermal drying system structure [1-3], indicating the technology method of using objective drying to achieve energy efficiency [4-6], it also develops the grain drying adaptive control system [7-8]. But the reliable water line detection is still one of the difficulties to achieve technology applications. Moisture content related to the physical and chemical properties of food, it is an important indicator to evaluate product quality. It is also one of the key parameters essential to achieve the drying process control and energy-saving. But there is still a lack of moisture measurement devices and techniques of accuracy and stability. Concerning the microwave, infrared, electrical and pressure method, the development line detector has been tried a lot [12]. The Common disadvantage of microwave, infrared and capacitive method is that the shape of grain, thickness, density and grain moisture distribution impact much to them [13-18]. With high dust environment and lower detection precision due to grain moisture fluctuation, the biggest problem of medium method is the measurement of sub-scattering properties of hydrogen instability. The changes of the relationship between the neutron count ratio and the volume of grain moisture content of food varies by species [19], different varieties of food is not the same in different times. Using neutron method, it must make the appropriate pre-processing for each batch of material measuring grain moisture content, the technology is not yet mature. Using neutron method, it must make the appropriate pre-processing for each batch of material measuring grain moisture content, the technology is not yet mature. Based on the resistance method, it develops the high-precision single grain moisture content of grain online detector [20], but reliable online sampling problems plaguing the application of technology. Grain drying system has many uncertainties, high temperature, high humidity, high dust, flow pattern fluctuations, causing great difficulties in impurity rate change detection accuracy and reliability. as for online measurement of specific technical issues, such as building oscillation frequency under flow conditions sensor [13], exploring the dielectric loss factor [21-22], improving plate structure [23-28], detection and control, it has a systematic study [29-31]. So far, the examples 
of reliable technology products online applications are scare. The detection accuracy and reliability are influenced much by food flowing fluctuation. Therefore, in this paper, a non-contact parallel design floating plates Line capacitance grain moisture measuring device, using positioning for filling, batch steady-state measurements, wireless communication line testing to ensure accuracy and reliability of detection equipment, and to verify production applications test.

\section{System Hardware Structure}

Grain capacitive Moisture online testing system configuration shown in Figure 1.

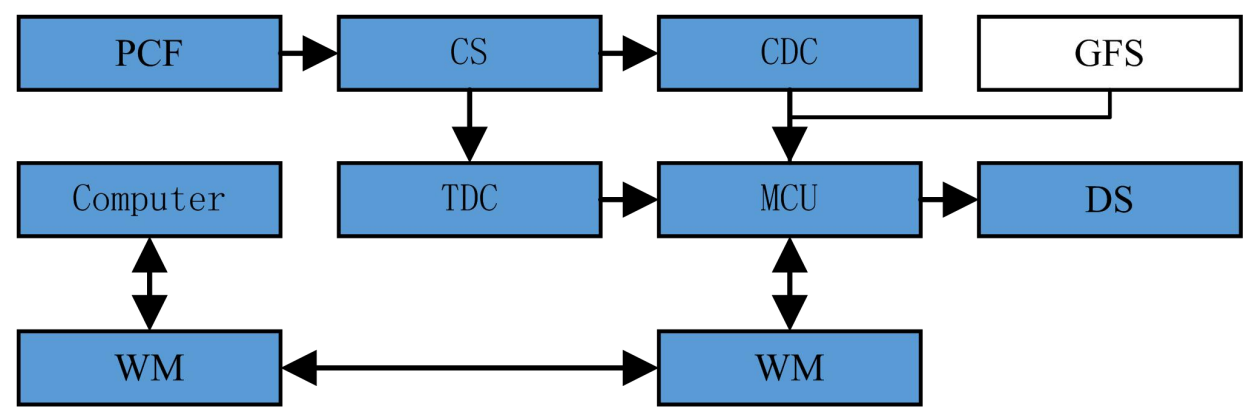

Fig.1 Composition of the system

Positioning charge feeder(PCF) installs near a grain dryer outlet, the outlet portion of food grain will fall and fill the feeders. Positioning charge feeder discharges roller to stably feed grain uniform capacitive sensors(CS) to ensure that the sensor food consistent porosity within the same water rates. Top capacitive sensor has proximity switches, when the grain fill sensor(GFS), proximity switches the output signal to the microcontroller (MCU), then MCU digital conversion circuit through a capacitor (CDC) and the temperature of the digital conversion circuit (TDC), recording the measured food capacitance and temperature values and calculating the corresponding water content. MCU row starts after the measurement of grain electromagnet, opens grain discharge side, the measured grain discharges sensor system(DS) into a single measurement. Measurements of process sensor transmit real-time computer users via the wireless module(WM), achieving remote monitoring.

\section{Online Moisture Detection Device}

Capacitive Sensor Structure. After ensuring the form of an electric field, the main factors affecting the measurement of the capacitance is the dielectric constant of the tested food, macro performance is polarization phenomena by measured dielectric constant food molecules in the electric field. Assuming that the capacitor measuring cavity is filled with food, the cavity distributes uniformly, you can measure the capacitance of the cavity of the food considered a mixture of dry air, water, carbohydrates. Air and carbohydrates are low loss dielectric, its constant with temperature change is small, at $50 \mathrm{kHz}$ frequency of approximately 1 and 3 to 5 ; water is high loss dielectric constant with temperature change, at $50 \mathrm{kHz}$ frequency, the dielectric constant of water $0 \sim 100^{\circ} \mathrm{C}$ was $55.88 \sim 78.9$. Because the dielectric constant of water is much larger than the other two substances, so the measured dielectric constant and its moisture content are directly related to food. The higher the moisture content is, the greater the dielectric constant is.

In order to obtain uniform and stable measurement of the electric field and reduce edge effects, the paper uses non-contact sheet structure parallel plates floating capacitance measure method. During the measure process, the grain doesn't touch the plates so that could prevent electrodes from electrochemical corrosion, but also improve the measuring accuracy of the grain capacitance under the high environment. The measuring area is wrapped with shielding later to be isolated from external electromagnetic interference. 


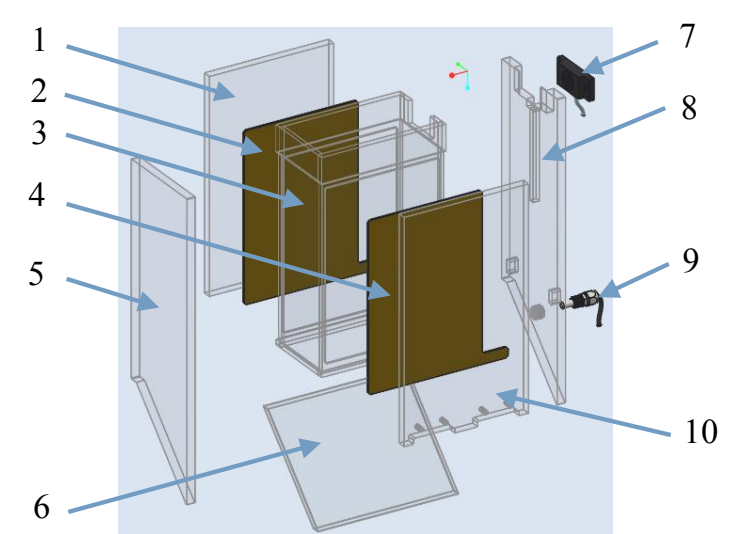

(a)

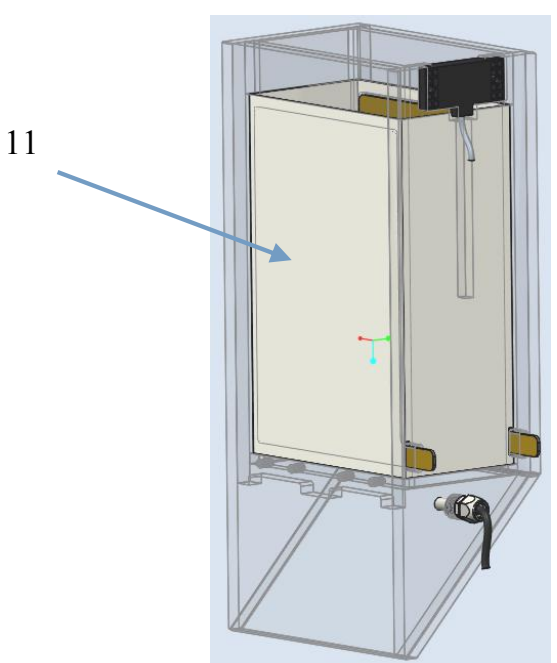

(b)

1、5、8、10. Protection board of sensor; 2、4. Electrode plates; 3. Container; 7. Material level sensor; 9. Digital temperature sensor; 6. Baseboard; 11. Shield

Fig.2 Structure of capacitive sensor

Figure 2 shows capacitable on-line detect grain sensor structure. Capacitor plate is $1.6 \mathrm{~mm}$ thick double-sided copper clad. These two plates are in the same size and shape, inner side plate capacitive measuring electrode and the opposite side connects the ground. The two capacitor plate are installed the surface of the measure cavity. The measured grain in the measure cavity is insulated with plate capacitor, as it is shown in Figure 2a. Attached a sided conductive aluminum foil between capacitor plates and ground to keep out external electromagnetic interference; the shield plate is slightly larger than the area of the portion having more equipotential ring, which could reduce the edge effect, shown in Figure $2 \mathrm{~b}$. The outside of the sensor installed the outer cavity protection board to protect the capacitor plates and the shield, and then to achieve waterproof and dustproof function.

The level sensor is mounted on the top of the sensor for detecting whether the food is filled in sensor cavity. The downside of the sensor is equipped with a digital temperature sensor for measuring food temperature, amending the influence of temperature on capacitance measurement.

Positioning filling material. Beyond the influence of temperature moisture on the grain capacitance, the bulk of the porosity also significantly affects the measurement capacitance. When the grain with the same moisture fall in measuring cavity in different ways, the voidage in the measuring cavity also will change, thus affect the measurement results. Because the voidage material is difficult to measure on-line, it is necessary to explore the effects and repeatability on voidage with different falling ways. The test sets 3 representative falling grain on-line mode types, Type 1 is Fixed Spill, and type 2 is Linetype Spill, and type 3 is Balanced Spill with the entire cross-section, in which Fixed Spill designs three spilled positions as 1 to 3; Linetype Spill designs 4 spilled positions respectively 4 to 7; Balanced Spill labels 8 spilled mode, as shown in Figure 3. Each spilled method divides into 3 kinds of spilled speed in which Fixed Spill's spilled speeds are 5, 10 and 20g/s; Linetype Spill's speeds are 10, 20 and $30 \mathrm{~g} / \mathrm{s}$; the speeds of Balanced Spill floating down are 25, 35 and $50 \mathrm{~g} / \mathrm{s}$. The moisture contents of corn samples are $13.3 \%, 15.1 \%$ and $17.6 \%$. Each test was repeated 20 times. Table 1 shows the measurement capacitance after being filled in capacitance cavity at 8 spilled modes. 


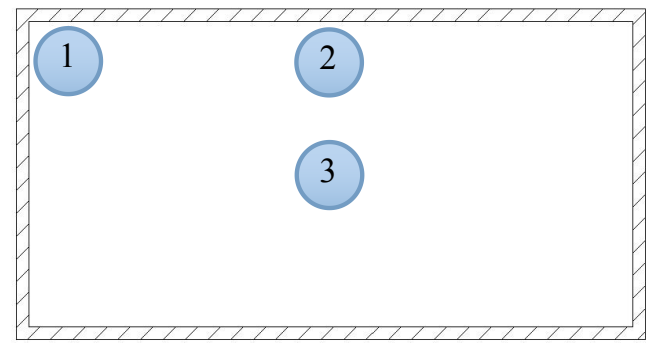

(a)

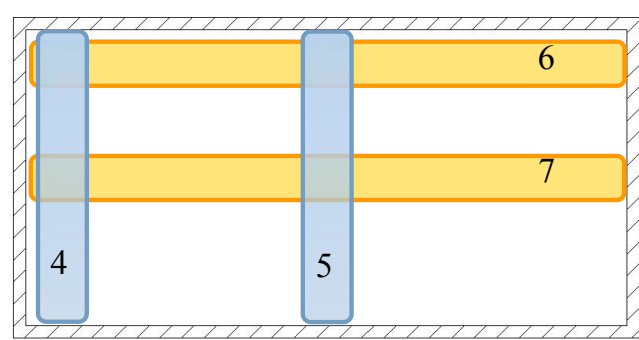

(b)

Fig.3 Top view of grain scatter forms

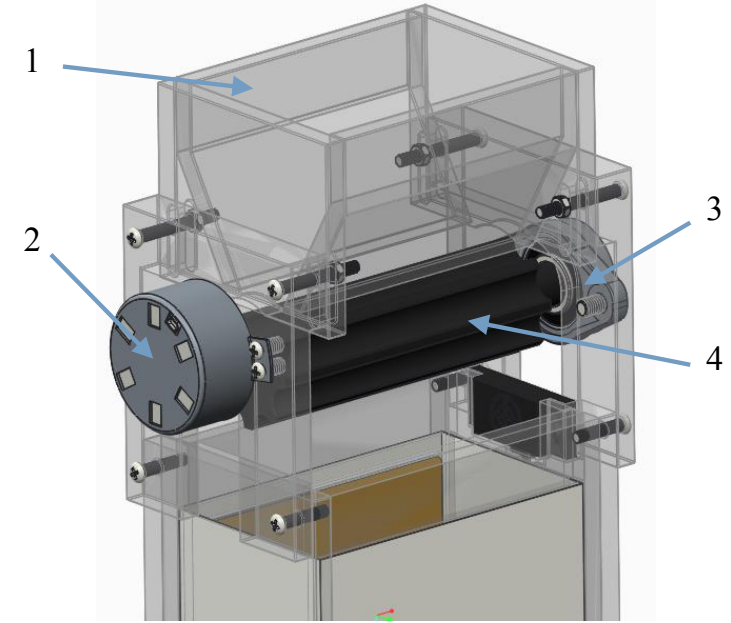

1. Hopper; 2. Electric motor; 3. Bearing; 4. Discharge roller

Fig.4 Structure of feed controller

As can be seen from Table 1, the water content of the corn was $13.3 \%$ in eight crisp ways, which measures capacitance changes from $8.8 \mathrm{PF}$ to $13.2 \mathrm{PF}$. The larger spill area is, the higher the measured capacitance is. The $15.1 \%$ and $17.6 \%$ corn's water content have the same characteristics and while the larger the spill area is; the moisture content of maize measuring capacitance changes will be more obvious. Therefore, the way of floating down has a significant influence on the measured capacitance. Compared the same spilled manner, but different spilled speed, and then measured corn's capacitance results could show that in the given spilled speed, the effects of changes of spilled speed on measuring capacitance are not obvious. It can be seen floating down significantly affects measurement of capacitance, while spilled position has few effects on measuring capacitance. Therefore, a reasonable measure for controlling food spilled into the cavity mode could avoid the effects of the online testing process on the accuracy of measuring and then crisp linear positioning charge feeder is designed. Figure 4 shows the positioning filling feeders which is mounted on the top of the cavity, Motor drives the discharge roller rotate grains in the hopper spill in uniform linear manner. Set the corn on-line measuring as example, the average spill rate is about $15 \mathrm{~g} / \mathrm{s}$.

Tab.1 Capacitance of different grain scatter forms

\begin{tabular}{|c|c|c|c|c|c|c|c|c|c|c|}
\hline \multicolumn{3}{|c|}{ spilled manner } & 1 & 2 & 3 & 4 & 5 & 6 & 7 & 8 \\
\hline \multirow{6}{*}{ 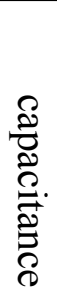 } & \multirow{3}{*}{$13.3 \%$} & speed 1 & $8.8 \pm 0.2$ & $8.8 \pm 0.1$ & $8.6 \pm 0.3$ & $10.0 \pm 0.4$ & $9.8 \pm 0.3$ & $11.9 \pm 0$ & $12.1 \pm 0.2$ & $13.2 \pm 0.5$ \\
\hline & & speed 2 & $8.8 \pm 0.1$ & $9 \pm 0$ & $8.9 \pm 0.2$ & $10.0 \pm 0.2$ & $9.9 \pm 0.1$ & $12 \pm 0.1$ & $12.2 \pm 0$ & $13.5 \pm 0.3$ \\
\hline & & speed 3 & $8.9 \pm 0.2$ & $8.8 \pm 0.1$ & $8.8 \pm 0.2$ & $10.0 \pm 0.3$ & $9.8 \pm 0.2$ & $12 \pm 0$ & $12.1 \pm 0.1$ & $13.1 \pm 0.4$ \\
\hline & \multirow{3}{*}{$15.1 \%$} & speed 1 & $10.1 \pm 0.2$ & $10.2 \pm 0.1$ & $10.0 \pm 0.1$ & $12.3 \pm 0.1$ & $12.3 \pm 0.3$ & $13.7 \pm 0.2$ & $13.8 \pm 0.1$ & $15.0 \pm 0.3$ \\
\hline & & speed 2 & $10 \pm 0.2$ & $10.1 \pm 0.2$ & $10.0 \pm 0.2$ & $12.2 \pm 0.3$ & $12.3 \pm 0.3$ & $13.7 \pm 0.2$ & $13.6 \pm 0.3$ & $15.1 \pm 0.2$ \\
\hline & & speed 3 & $10.1 \pm 0.2$ & $10.1 \pm 0.2$ & $10.0 \pm 0.1$ & $12.2 \pm 0.1$ & $12.1 \pm 0.2$ & $13.7 \pm 0.2$ & $13.7 \pm 0.2$ & $15.0 \pm 0.4$ \\
\hline \multirow{3}{*}{$\vec{\partial}$} & \multirow{3}{*}{$17.6 \%$} & speed 1 & $13.8 \pm 0.1$ & $14.0 \pm 0.2$ & $13.9 \pm 0.1$ & $15.8 \pm 0.2$ & $15.9 \pm 0.1$ & $16.9 \pm 0$ & $16.9 \pm 0.2$ & $18.8 \pm 0.3$ \\
\hline & & speed 2 & $13.7 \pm 0.2$ & $14.0 \pm 0.1$ & $14.0 \pm 0.2$ & $16 \pm 0$ & $15.7 \pm 0.2$ & $16.9 \pm 0.1$ & $17 \pm 0.2$ & $18.8 \pm 0.3$ \\
\hline & & speed 3 & $13.7 \pm 0.1$ & $14.0 \pm 0.2$ & $14.8 \pm 0.2$ & $15.8 \pm 0.2$ & $15.7 \pm 0.2$ & $16.9 \pm 0.1$ & $16.7 \pm 0.4$ & $19 \pm 0.4$ \\
\hline
\end{tabular}


Grain Discharge Mechanism. During on-line testing process, in order to obtain a stable, accurate and repeatable capacitance, the grain in the measuring cavity is collected and measured in batch steady way. The sensor measures about $800 \mathrm{~g}$ grains for each measurement, and each measurement needs about $1.5 \mathrm{~min}$ to finish. Compared with $6-7 \mathrm{~h}$ 's grain drying process, it can be regarded as the real-time measurement, and no measuring lag exist. After the measurement, it empties the measured grains rapidly to ensure that new measuring grains will not mix with measured grains. Therefore, it is necessary to design a reasonable grain discharge mechanism so that agencies can be efficient, stable and in reliable operation.

Using similarity theory, measurement cavity may be assimilated as a dark warehouse. From the granary effect theory proposed by Janssen, we find that positive pressure and lateral pressure on the bottom side of the barn will not increase linearly with the fill level, but with the increasing of height, the amplitude slows down. When the grain reaches at a certain height, the pressure of the bottom side will tend to a saturation value. During the whole process, the lateral pressure is far less than the positive side [33].

Based on the above principles, in order to ensure the flexible of grain discharge, it is necessary to reduce the pressure during the measurement of grain discharge mechanism, so opening grain discharge mechanism from the lateral side is designed. In the stationary state, the spring has a certain pre-tension to ensure discharge door cannot be opened by lateral forces acting, as shown in Figure 5a. When grain discharge, the solenoid is energized, and pushes the pivot arm against the spring tension to open the discharge door, as shown in Figure $5 \mathrm{~b}$. After $4 \mathrm{~s}$, the grain is emptied and solenoid will be automatic power-off, and then discharge gate will get back with the spring elastic reset.

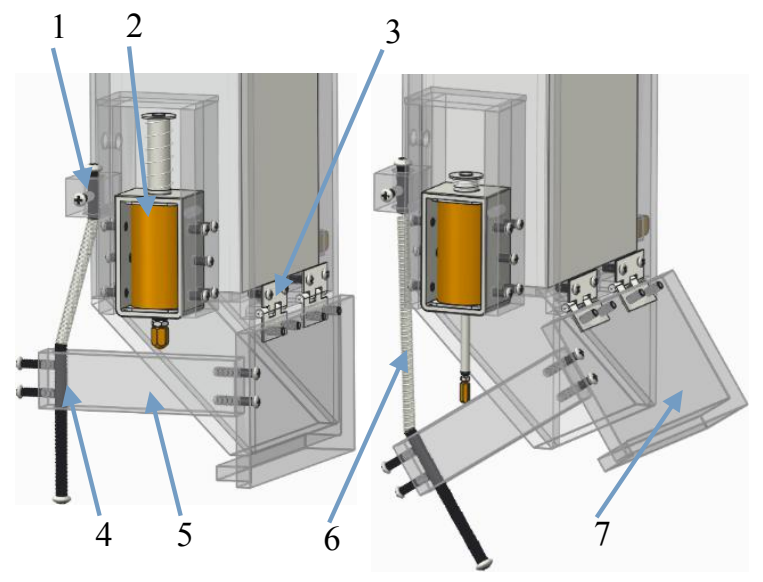

(a)

(b)

1. Spring fixed end 2. Electromagnet 3. Hinge 4. Spring adjusting screw 5. Tumbler 6. Spring 7. Discharge baffle plate

\section{Fig.5 Structure of grain discharge}

Micro-capacitance Measuring Circuit. When the cavity is empty, capacitance is $0.2 \mathrm{PF}$. When filled with food, the capacitance is $5 \sim 35 \mathrm{PF}$. In order to obtain the high accuracy moisture data, measuring circuit resolution must be less than $0.1 \mathrm{PF}$, which uses precision the traditional method and the resonant impedance method are difficult to achieve. Regarding AD7745 capacitance-digital converter chip as a micro-capacitance detection element has been gradually widely used. AD7745 capacitance's measurement ranges from 0 to $21 \mathrm{PF}$. With the use of capacitive ratio method, the capacitance may expand to $0 \sim 120 \mathrm{PF}$. This paper used AD7745, its expansion circuit and microcontroller build grain capacitive on-line to detect small capacitance measurement circuit. The hardware principles are shown in Figure 6. The controller uses an enhanced measurement circuit 51 as STC12C5620AD, external crystal is $22.1148 \mathrm{MHz}$, which has a high operation speed and stability. The microcontroller and the AD7745 communicate through the IIC interface. 
Circuit measuring principle uses floating method for reducing the sensor plates to the parasitic capacitance to ground and leakage to the ground. Two capacitor plate leads use SYV-75-2-1 coaxial cable, while cable shielding layer and the sensor are connected and grounded to further eliminate the parasitic capacitance and the coupling capacitance between the leads.

For the performance test of the measurement circuit, please refer to GB/T 13978-2008 standards and combine with space-variant capacitance and TH2821B LCR digital bridge to calibrate circuit. The calibration result shows that the circuit is $0.01 \mathrm{PF}$, and the accuracy is $\pm 0.1 \mathrm{pF}$, and the measurable ranges from 0 to $85 \mathrm{PF}$, which could satisfy the measurement requirements.

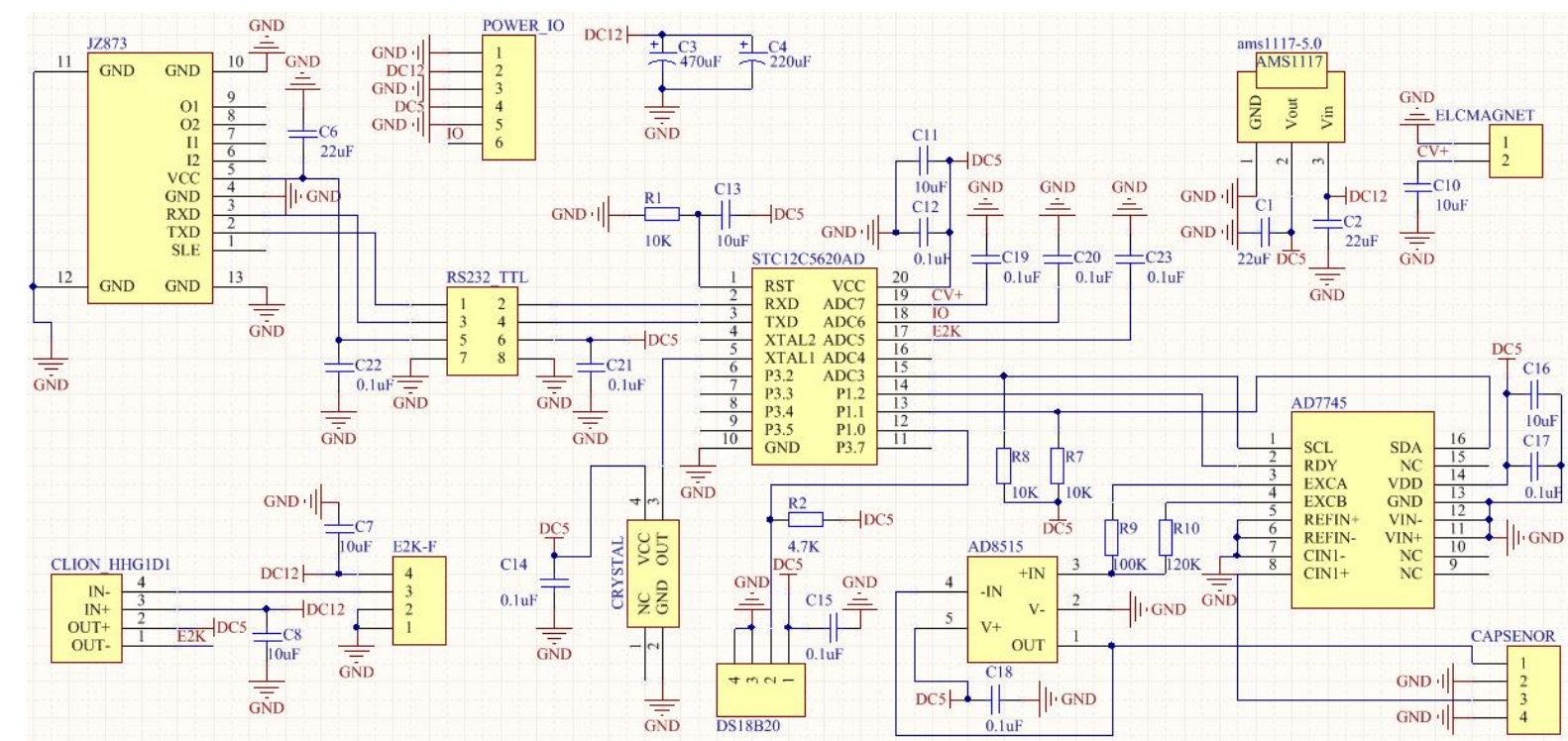

Fig.6 Hardware principle diagram of micro-capacitance gauging circuit

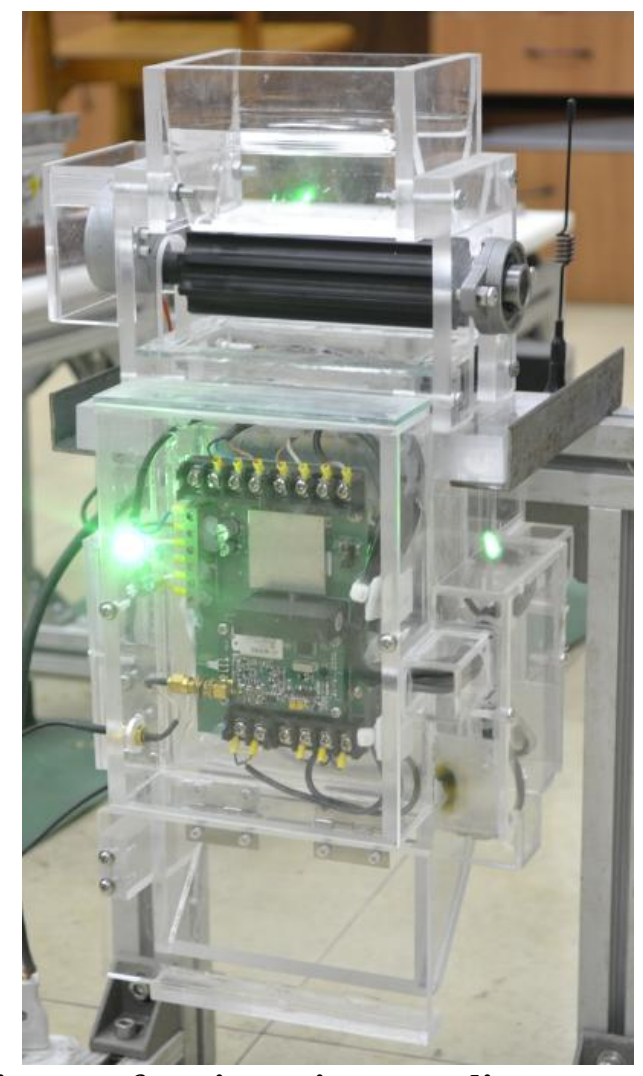

Fig.7 Thepicture of grain moisture online measuring sensor 
Wireless communication network. In order to ensure the real-time, accurate and stable communication between the water on-line device and the computer, avoid the influence of the bad environment of the drying field on the data communication, and reduce the installation period and the cost of the equipment, we designed a wireless communication network, which is suitable for moisture content in this paper. Sensor ends adopt JZ873 wireless data transmission module, which uses the transparent transmission way through the serial port and the single chip microcomputer realizes the data to send and receive; Computer side adopts USB JZ874 wireless data transmission module. The transmission frequency between the two modules is $433 \mathrm{MHz}$, the baud rate is $9600 \mathrm{BPs}$, using 3.5 DB sucker antenna, wireless transmission distance not less than $2000 \mathrm{~m}$, to meet the requirements of grain drying data transmission distance.

Grain moisture on-line detection device and computer wireless communication using ASCII Modbus communication protocol. The protocol includes address code, function code, data range and LRC verification, to achieve the communication between multiple devices.

Protection design. A large amount of water vapor is discharged during the operation of the grain drying equipment. The grain is rubbing against each other, and a large amount of dust is produced under the action of the fan and the lifting machine in the process of drying grain. Therefore, in reference to the GB 4028 - 2008 of the power of the sensor, control circuit and actuator for waterproof and dust treatment, to achieve the IP64CM level. Sensors is constantly subject to grain erosion in the detection process in the absence of protection, it will be severely worn in a short time, So the glass with a thickness of $1 \mathrm{~mm}$ must be pasted in the external surface of the grain moisture on-line detection device and the inner wall of capacitance measuring vessel.

\section{Test}

Calibration. The calibration test of corn drying capacitance, temperature and moisture relationship was held in the growing corn field of corn drying capacitance Niuwanggong village Laoqitai town Qitai county Changji state Xinjiang procince in October 2013. The capacitance sensor is installed in the lower of the $200 \mathrm{t}$ continuous corn drier, as shown in picture 8 . The dry raw material is "new jade 10", the initial moisture content of corn is about $20 \%$, the impurity rate is $5 \%$, the moisture content of drying target is $12.5 \%$. Calibration test of 3 columns of corn. In order to obtain the corresponding relationship between the water and the capacitance of the corn, the dryer is dry in a circular way. First tower dryer hot air temperature is $(70+5) \mathrm{C}$, second tower dryer hot air temperature is $(60+5)$ degrees $C$, third tower dryer hot air temperature is $(50+5)$ degrees $C$. In the process of grain drying, every 30min is sampled at the bottom of the sensor, in accordance with the GB/T 5497 - 1985 standard, the samples were crushed, Measuring moisture content with constant time and temperature oven method for $(130+2)$ min and $40 \mathrm{C}$. The measured results were compared with the corn capacitance measured by capacitive grain moisture on line detection device, to get the corresponding relationship between water content and capacitance was obtained at different temperatures.

As multivariate nonlinear regression for real time online detection data, we get the

$$
M=a_{0}+a_{1} X_{c}+a_{2} X_{T}+a_{3} X_{c} X_{T}+a_{4} X_{c}^{2}+a_{5} X_{T}^{2} \text { (1) }
$$

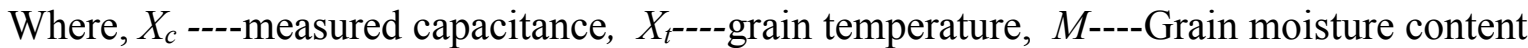

Coefficient of determination $R^{2}=0.984$, in which, $\mathrm{a}_{0}=11.1941, \mathrm{a}_{1}=2.1397, \mathrm{a}_{2}=-0.7196, \mathrm{a}_{3}=0.0367$, $\mathrm{a}_{4}=-0.0879, \mathrm{a}_{5}=0$.

Verification. The relationship between the capacitance and water content and temperature of the corn is written into the computer human interface in the formula (1), and the computer calculates grain moisture content according to the temperature and capacitance measured by the moisture on line detection device. The air temperature fluctuations are in the range of $15 \sim 50 \mathrm{DEG} \mathrm{C}$, and the relative humidity is $80 \% \sim 100 \%$ installation position of capacitive moisture on line detection device in test process. Calculate the moisture content in corn on line and in real-time, sample at the bottom of the sensor, and measure the actual moisture content of the corn samples with the oven method. Compare the measured value of On-line device with that of the oven method. As shown in picture 9. 


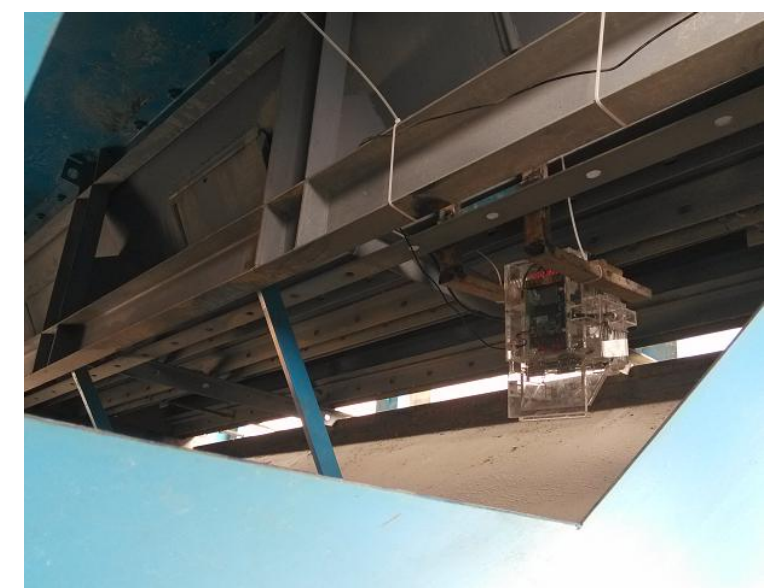

Fig.8 Equipment installation diagram

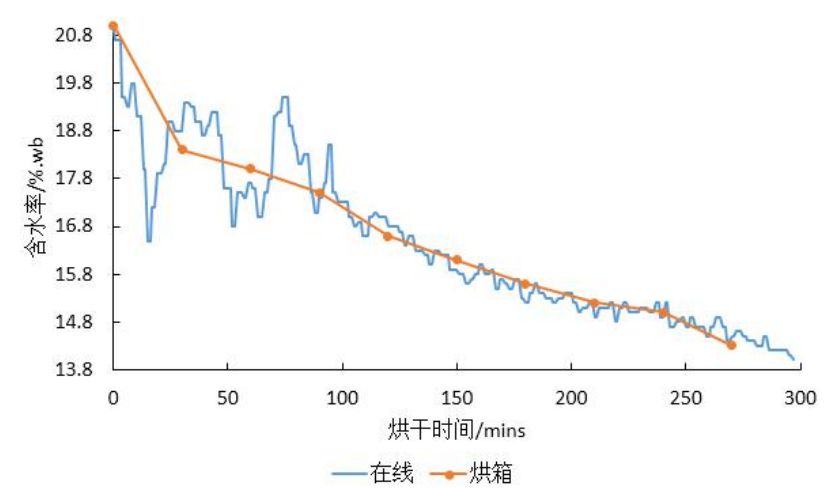

Fig.9 Comparison of moisture contents measured by self-designed grain moisture online measuring device and standard oven drying method

As we can see from picture 9, the measured water content and the actual water content of the gap is not. Compared with the actual water content, the absolute error of water cut measurement between $-0.4 \% \sim 0.4 \%$, the average absolute error is $0.23 \%$.

\section{Conclusions}

(1) Use the non-contact measurement of parallel floating capacitance method to test the grain capacitors, and the grain is measured in batch. The grain is in a steady state in the measurement process, therefore, the capacitance has a higher stability.

(2) Positioning charging device ensures the uniformity of grain filling positioning and improves the accuracy and repeatability of measurement sensor.

(3) The use of wireless communication, realizes the communication between controller and online detection device, to avoid the loss of some uncertain factors on the detection reliability.

(4) Fill the material by positioning, measure it in a steady state in batches, and the wireless communications ensure the accuracy and reliability of online testing. At a temperature of $15 \sim 50{ }^{\circ} \mathrm{C}$ and under the $21 \%$ dynamic conditions, the maximum deviation is less than the on-line detection of relative humidity in $80 \% \sim 100 \%$, and the corn moisture content is in the range of $14 \% \sim 0.4 \%$.

\section{Acknowledgements}

This work was financially supported by The Research Fund for the Doctoral Program of Higher Education of China (20114404110021, 20060564003). 


\section{References}

[1] Li Changyou, Mai Zhiwei, Fang Zhuangdong. Analytical study of grain moisture binding energy and hot air drying dynamics[J].Transactions of the CSAE, 2014, 30(7): 236-242.

[2] Li Changyou, Ma Xingzao, Fang Zhuangdong, etal. Thermal energy structure of grain drying by analytical method[J]. Transactions of the CSAE, 2014, 30(9): 220-228.

[3] Li Changyou. Exergy evaluation theory of hot air drying system for grains[J]. Transactions of the CSAE, 2012, 28(12): 1-6.

[4] Li Changyou, Zhang Ye, Mai Zhiwei. Design and experimental study of dryer for high moisture grain storage[J]. Transactions of the Chinese Society for Agricultural Machinery, 2014, 45(4): 231-235.

[5] Li Changyou, Fang Zhuangdong. Analytical model of high moisture content paddy in multistage countercurrent drying and tempering $[\mathrm{J}]$. Transactions of the Chinese Society for Agricultural Machinery, 2014, 45(5): 210-215.

[6] Li Changyou, Mai Zhiwei, Fang Zhuangdong, etal. Design and test of seed circulation drying system[J]. Transactions of the Chinese Society for Agricultural Machinery, 2014, 45(6): 242-24.

[7] Li Changyou, Ban Hua, Shen Wenhao. Self-adaptive control system of grain drying device[J]. Drying Technology, 2008, 26(11): 1351-1354.

[8] Li Changyou, Ban Hua. Self-adaptive control system of grain drying device based on deep bed analytical ventilating drying theory[J]. Transactions of the CSAE, 2008, 24(4): 142-14.

[9] Zhao Baojun, Qu Chunbo, Li Yang. Improvement of QB475 Infrared Moisture Instrument [J]. Journal of Electronic Measurement and Instrument, 1999, 13(1): 67-70.

[10] Fu Haidong. The design and research on grain moisture content on line measuring system based on pressure[D]. Changchun: Jilin Agricultural University, 2012.

[11]Zhang Xi. Design of microwave moisture content instrument[D]. Shenyang: Shenyang University of Technology, 2007.

[12]Dong Yiwei. On the online determing method of water content of grain based on non-uniform electric field[D]. Nanjing: Nanjing Agricultural University,2007.

[13] Mai Zhiwei, Li Changyou, Zhang Ye, etal. Application of wireless data transmission technique in drying equipment[J]. Advanced Materials Research, 2012, 422: 262-26.

[14] Mai Zhiwei. Online moisture testing system for grain[D]. Guangzhou: South China Agricultural University, 2011.

[15]Qiu Yu. Design of portable capacitive grain moisture detector based on flat panel structure[D]. Guangzhou: South China Agricultural University, 2013.

[16] Yang Kang, Guo Haifeng, Xu Yankai. Solving the axial edge effect in designing capacitance sensor[J]. Chinese Journal of Scientific Instrument, 2002, 23(3): 115-11.

[17] Zhang Shuzhen, Ba Weiguo, Xu Baojiang, etal. Study and Manufacture of Porosity Determining Apparatus of an Unconsolidated Mass of Materials[J]. Transactions of the Chinese Society for Agricultural Machinery, 1996, 12(1): 187-191.

[18] Yang Liu, Yang Haoming, Dong Lanlan. Development of acoplanar electrode capacitance moisture sensor[J]. Transactions of the Chinese Society for Agricultural Machinery, 2010, 41(1): 71-8. 
[19] Yang Yueqian, Wang Jianping, Wang Chengzhi. Study on line measurement of grain moisture content by neutron gauge[J]. Transactions of the CSAE, 2000, 16(5): 99-10.

[20] Li Changyou. Design and experiment of online moisture metering device for paddy drying process[J]. Transactions of the Chinese Society for Agricultural Machinery, 2008, 39(3): 56-59.

[21] Cheng Weidong, Bo Xueyuan, Wang Xiangyou, etal. An on line measurement and monitoring system of grain moisture during drying process[J]. Transactions of the Chinese Society for Agricultural Machinery, 2000, 31(2): 53-55.

[22] Li Yede. Method for measuring grain moisture content based on dielectric loss tangent[J]. Transactions of the CSAE, 2006, 22(2): 14-17.

[23] Teng Zhaosheng. An intelligent instrument for quick measurement of cereal moisture content based on self-adaptive fusion of weighted data[J]. Transactions of the Chinese Society for Agricultural Machinery, 1999, 30(6): 64-6.

[24] Li Zaigui, Yin Lijun, Gao Zhenjiang, etal. Effects of moisture conditions of grain on inductance of high frequency coil[J]. Transactions of the CSAE, 2004, 20(3): 149-152.

[25] Yang Liu, Mao Zhihuai, Dong Lanlan. Development of plane polar probe of capacitive grain moisture sensor[J]. Transactions of the CSAE, 2010, 26(2): 185-18.

[26] Ma Chao. Research on capacitance detecting method and the detecting device for grain moisture[D]. Guangzhou: South China Agricultural University, 2008.

[27] Wang Xudong. The research and design for ARM $\mu$ Clinux data collection system of grain drying[D]. Guangzhou: South China Agricultural University, 2008.

[28] Teng Zhaosheng, Tang Ruiming, Qi Chaofu. Design of a Type of Insertion Smart Sensor with Low-power for Measuring temperature \& Moisture Content of Grain [J]. Journal of Electronic Measurement and Instroment, 2000, 14(3): 49-53.

[29]Ban Hua. Grain moisture on line measurement and expert system of concentrate drying[D]. Guangzhou: South China Agricultural University, 2006.

[30] Teng Zhaosheng, Ning Lewei, Zhang Haixia, etal. On line measurement system of grain dryer for monitoring moisture content[J]. Transactions of the CSAE, 2004, 20(5): 130-133.

[31] Cui Qingliang, Guo Yuming, Zheng Decong. Design and test of on line measurement system for the moisture content of the freeze drying materials[J]. Transactions of the Chinese Society for Agricultural Machinery, 2008, 39(4): 91-96.

[32] Zhang Shuzhen, Ba Weiguo, Xu Baojiang, etal. Study and Manufacture of Porosity Determining Apparatus of an Unconsolidated Mass of Materials[J]. Transactions of the Chinese Society for Agricultural Machinery, 1996, 12(1): 187-191.

[33] Zhang Jiakang, Huang Wencui. A new method for pressure calculation in silos[J]. China Civil Engineering Journal, 2000, 33(5): 24-28. 